Auf die Reaktion der »anspruchsvollsten Anspruchsgruppen « und Standardsetzer war das Unternehmen gespannt. Die weitgehende »Funkstille« im Bereich der Umwelt- und Verbraucherorganisationen war deshalb auf den ersten Blick besonders enttäuschend. Allerdings nur auf den ersten Blick. Denn für die - bisherige - Zurückhaltung gibt es eine ganze Reihe plausibler Gründe:

- Das auf beiden »Seiten « lange vorherrschende Lagerdenken ist nur schrittweise zu überwinden.

- Es gibt - ebenfalls auf beiden Seiten - die durchaus berechtigte Angst, von der jeweils anderen Seite vereinnahmt und über den Tisch gezogen zu werden - mit der Folge eines Profil- oder gar Identitätsverlustes. Die klare Abgrenzung beider Seiten hat ja auch den sehr positiven Effekt, kreatives Potential zu erzeugen, was durch einen zu engen Dialog oder gar Kooperation leicht verwässert werden kann.

- Sowohl Unternehmen als auch Umwelt- und Verbraucherverbände stehen im Wettbewerb. Eine klare Positionierung zum Wettbewerber ist deshalb (überlebens-)wichtig; ein zu »grünes « Image kann für Unternehmen, ein zu »wirtschaftfreundliches« für Anspruchsgruppen von erheblichem Nachteil sein.

- Die »Themenkonjunkturen« beider Seiten verlaufen nicht parallel, sondern versetzt: Die Existenzberechtigung und der $»$ gesellschaftliche Wert « von Umwelt- und Verbraucherverbänden u. a. Anspruchsgruppen besteht ja zu einem großen Teil darin, schneller zu sein als Gesellschaft, Politik, Wirtschaft und oft auch Wissenschaft. Sie schaffen oder besetzen zukünftige Themen, die dann, wenn sie sich als gesellschaftliches Thema durchgesetzt haben, von den anderen gesellschaftlichen Akteuren erst en detail »abgearbeitet«. werden müssen - und in dieser Zeit schaffen die Anspruchsgruppen neue Themen. Der Zeitraum, in dem alle Seiten parallel diskutieren, ist damit relativ kurz.

- Schließlich gibt es die sehr banalen, aber um so schlagenderen knappen Ressourcen. Dia$\log$ und Kooperation kosten beide Seiten Zeit und Geld und das ist auf beiden Seiten knapp, vor allem aber bei den Anspruchsgruppen. Effizientes Ressourcenmanagement wird deshalb versuchen, die Wege so kurz wie möglich und den Dialog so konkret wie möglich zu machen: Der Dialog über ein konkretes Problem oder Projekt zwischen Partnern an einem Ort ist um Größenordnungen effizienter als ein allgemeiner Dialog zwischen zwei Partnern, die $500 \mathrm{~km}$ voneinander entfernt sitzen.

\section{Resuimee}

Die geschilderte positive Reaktion von Externen wirkt sich natürlich positiv auf die interne Ent- wicklung aus und umgekehrt. Zusammen haben diese Entwicklungen in der Tat das Potential, zu einem wenigstens teilweise »selbstorganisierenden Prozeß « zu werden, wobei ein - guter Umweltbericht sicher nicht alleinige Ursache ist, aber doch eine wichtige Katalysatorfunktion erfüllen kann.

Für eine dauerhaft positive Entwicklung letztlich entscheidend ist jedoch immer der nächste

1) Kraft Jacobs Suchard Deutschland, Umweltbericht 1991 -1995, erhältlich über: Kraft Jacobs Suchard Deutschland, Environment \& Associations, Langemarckstraße 4-20, 28199 Bremen; Tel. : 0421 / 599 - 3867, Fax: -3899). Im folgenden beziehen sich Seitenzahlen in Klammern auf diesen Bericht.

2) Eine klare Ausnahme von der Ausnahme ist der BUND, insbesondere der Landesverband Bremen, mit dem seit über drei Jahren ein sehr fruchtbarer Dialog besteht.

3) Vor allem die Definition von Grundpositionen und Zielen sowie der Aufbau eines ÖkoControlling-Systems und die Erstellung einer Input-Output-Analyse.

4) Vgl. Clausen, J.; Fichter, Klaus: Wissen-
Umweltbericht: Er bestimmt die interne und externe Diskussion, die Ziele und die konkreten Maßnahmen, bevor er veröffentlicht wird. Der zweite Umweltbericht von Kraft Jacobs Suchard Deutschland erscheint 1996 - das Unternehmen befindet sich $\mathrm{ab}$ sofort in der entscheidenden Phase.

Heiko Richert, Bremen

schaftlicher Endbericht Umweltweltberichterstattung

5) Erreicht wurden knapp 284 von 500 Punkten (entspr. 57\%) und wäre (zum Zeitpunkt der Erstellung des Gutachtens: Januar 1994) im IÖWRanking der fünftbeste deutsche Umweltbericht gewesen. Das Gutachten und die Bewertung sind nach Meinung des Unternehmens fair und zutreffend. Die festgestellten Mängel sollen möglichst im nächsten Bericht (1996) behoben werden.

6) imug - Institut für Markt - Umwelt - Gesellschaft u. a. (Hg. ), Der Unternehmenstester. Die Lebensmittelbranche, Reinbek bei Hamburg 1995 , S. 187 f.

\title{
Umwelterklärungen als Instrument dialogorientierten Managements - Anforderungen der Öffentlichkeit
}

Das Verhältnis der Unternehmen zur Öffentlichkeit ist in der Betriebswirtschaftslehre traditionell ein Teilgebiet der Marketinglehre. Public Relations umfassen die Kommunikationsbeziehungen zwischen einem Unternehmen und der nach Teilgruppen gegliederten Öffentlichkeit. Gleichwohl hat die Ausrichtung auf die Anforderungen der nichtmarktlichen Anspruchsgruppen in den Betrieben noch keineswegs den Stellenwert der Kundenorientierung erreicht. Noch immer ist die PR-Abteilung im wesentlichen als Stabsstelle der Unternehmensleitung zugegliedert; ohne Durchgriff und zumeist auch ohne Einfluß auf unternehmenspolitische Entscheidungen und letztlich auf die reaktive Erhaltung von Handlungsspielräumen kapriziert (1). Die ernsthafte Kommunikation mit umweltbezogenen Anspruchsgruppen ist heute im besten Fall Stiuckwerk, das aus dem Interesse einzelner aufgeschlossener Manager entsteht. Umgekehrt bleibt auch das Interesse der gesellschaftlichen Anspruchsgruppen am Umweltverhalten der Unternehmen einzelfallorientiert und ohne systematische Einbindung.

Angesichts der negativen Erfahrungen aus der Geschichte der Sozialbilanzierung (2) und dem offenkundigen Widerwillen gegen öffentliche Transparenz auch bei ansonsten umweltpolitisch aktiven Unternehmen ist es ein spannendes gesellschaftliches Experiment, ob die Umwelterklärung im Rahmen der EU-Ökoaudit-Verordnung die Basis für eine systematische, dialogorientierte betriebliche Umweltpolitik bilden kann.

\section{Informationsbedürfnisse der gesellschaftlichen Anspruchsgruppen}

In der EU-Verordnung zum Umweltmanagement und zur Umweltbetriebsprüfung kommt der Transparenz der Unternehmen gegenüber der Öffentlichkeit eine herausgehobene Bedeutung zu. Die Umwelterklärung stellt dazu das zentrale Element der Verordnung dar. Auf indirektem Weg sollen neue Informationstatbestände und neue Informationsflüsse erzeugt werden, die ihrerseits Marktkräfte im Sinne des Umweltschutzes induzieren (3).

Soll die Umwelterklärung ihre Aufgabe erfüllen, so ist zu berücksichtigen, welche Informationsbedürfnisse die gesellschaftlichen Anspruchsgruppen als Adressaten der Umwelterklärung haben. Voraussetzung ist, daß es sich um berechtigte, sinnvolle Ansprüche handelt. Probleme ergeben sich dabei aus dem Umstand, daß der Adressatenkreis heterogen sein kann, sehr unterschiedliche Interessen verfolgt und entsprechend unterschiedliche Informationsan- 
sprüche stellt. Hinzu kommt, daß oftmals schwer zu bestimmen ist, welche Angaben zu welchen Assoziationen bei den Zielgruppen führen.

Im Vordergrund stehen bei der Umwelterklärung durch deren Standortbezug Anwohner, Bürgerinitiativen und kommunale Behörden. Bei der Erklärungserstellung ist dementsprechend danach zu fragen, welche Informationsbedürfnisse die genannten Adressaten formulieren. Dabei wird sich unter anderem sehr schnell herausstellen, daß sich die Forderungen der Anwohner durchaus unterscheiden können von den Informationsverpflichtungen, wie sie durch das geltende Umweltrecht gegenüber den Behörden bestehen. Eine zusammenfassende Aufbereitung von Informationen, die aufgrund anderer gesetzlicher Vorgaben sowieso bereitzustellen sind, kann sicherlich wertvolle Hinweise liefern, reicht aber nicht aus. Zwar sind die Anforderungen an die Verständlichkeit von Unterlagen, die in Genehmigungsverfahren für Industrieanlagen vorgelegt werden müssen, durch die einschlägigen verwaltungsrechtlichen Verfahrensvorschriften (4) relativ hoch angesetzt. So müssen z. B. allgemein verständliche Kurzbeschreibungen von zu genehmigenden Industrieanlagen und Zusammenfassungen von Umweltverträglichkeitsuntersuchungen (UVU) dem Genehmigungsantrag beigefügt werden. Das Manko dieser Unterlagen liegt aber darin, $\mathrm{da} ß$

- diese Informationen nach Abschluß der Genehmigungsverfahren nicht mehr allgemein zugänglich sind,

- die Unterlagen bestimmungsgemäß

a) allein den zu genehmigenden Anlagenbereich betreffen, nicht den gesamten Standort, dessen Anlagen möglicherweise durch eine Vielzahl von Genehmigungen zugelassen wurden,

b) und jeweils nur den spezifisch rechtlichen Sektor erforderlicher paralleler Genehmigungen $z$. B. aus dem Immissionsschutzrecht oder Wasserrecht betreffen.

Eine ernsthaft gemeinte Kommunikationspolitik wird sich vielmehr damit auseinandersetzen müssen, welche Kommunikationsbedürfnisse im einzelnen bei den Anwohnern bestehen und dazu ggf. auf Instrumente der empirischen Sozialforschung zurückgreifen.

Anders als die Informationsbedürfnisse im kommunalen und regionalen Bereich werden sich bei der überregionalen Öffentlichkeit, die sich z. B. in Umweltschutz- und Verbraucherverbänden wiederfindet, auch Fragen nach den Umweltwirkungen der Produkte durch Rohstoffgewinnung, Produktion, Nutzung und Entsorgung eine Rolle spielen. Desweiteren werden Fragen danach gestellt werden, in welchen ökologischen Zusammenhängen die verschiedenen Standorte eines Unternehmen stehen; beispielsweise ob bei Standorten im Ausland dieselben Umweltstandards angelegt werden wie im In- land oder aber ob ein Umweltdumping in Ländern mit vergleichsweise niederigen Umweltstandards stattfindet.

Die Verordnung schreibt einen standortspezischen Bericht vor; dies ist für eine auf Zielgruppen bezogene Umweltkommunikation allein nicht ausreichend: Sie sollte den Interessen der unterschiedlichen Anspruchsgruppen des Unternehmens gerecht werden, so daß neben dem auf Anwohner/Bürgerinitiativen und kommunale Behörden zugeschnittene Standortbericht zumindest ein zusammenfassender Konzernbericht notwendig erscheint.

\section{Grundsätzliche Anforderungen an eine gute Umwelterklärung}

Die Umwelterklärung hat eine Informationsund Rechenschaftsfunktion gegenüber ihren Adressaten. Allerdings ist in der EU-Verordnung eher generalklauselartig und wenig detailliert festgeschrieben, welche Bestandteile eine Umwelterklärung beinhalten soll. Vorgaben oder Hilfestellungen zur Berichterstattungstiefe sind nicht enthalten. Damit bleibt es auf den ersten Blick den an der Regelung teilnehmenden Unternehmen überlassen, Breite und Tiefe der offengelegten umweltbezogenen Informationen selbst zu bestimmen; ein Umstand der gegenwärtig bei den Unternehmen, bei potentiellen Umweltgutachtern, aber auch bei Umweltverbänden Verunsicherung hervorruft. Bei Fehlen jeglicher Standards werden die Ausgestaltungen von Umwelterklärungen derart unterschiedlich ausfallen müssen, daß Vergleichsmöglichkeiten den Adressaten schwerlich möglich sein werden.

Gleichwohl darf der Umweltgutachter die Umwelterklärung nur dann für gültig erklären, wenn sie sich «als genau, hinreichend detailliert und mit den Anforderungen des Systems vereinbar erweist« (5). Im Bereich der monetären Rechnungslegung von Unternehmen, d. h. Jahres- und Konzernabschlüssen, wurden für Formalanforderungen Rahmengrundsätze entwickelt (6). Um die Zielsetzung von Umwelterklärungen erfüllen zu können, bedarf es im Bereich der umweltbezogenen Berichterstattung eines Äquivalents, zumal sich dazu im Verordnungstext unmittelbare Anknüpfungspunkte finden lassen (7). Die Ableitung sog. Grundsätze ordnungsgemäßer Umweltberichterstattung kann mit Hilfe der hermeneutischen Methode geleistet werden. Die Einzelvorschriften sind so zu interpretieren, daß als Grundlage für die Interpretation der Vorschriften nicht nur deren Wortlaut und Wortsinn, sondern ebenso der Bedeutungszusammenhang innerhalb des Gesetzes, die Entstehungsgeschichte sowie die objektiv-teleologisch ermittelten Zwecke der Umwelterklärung dienen.

Als Grundsätze einer ordnungsgemäßen Umweltberichterstattung lassen sich unter Heranziehung der Vorgaben der EU-Verordnung folgende Grundsätze ableiten:
- Grundsatz der Vollständigkeit:

In der Umwelterklärung sind alle für den Standort bedeutsamen Umweltfragen zu berücksichtigen. (8) Welche Informationen als relevant einzustufen sind, richtet sich nach den Informationsinteressen der Umwelterklärungsadressaten und nicht nach der informationspolitischen Intention des Unternehmens.

\section{- Grundsatz der Richtigkeit:}

Die Angaben der Umwelterklärung müssen zuverlässig und damit wirklichkeitsgetreu erfolgen. Die Forderung nach Richtigkeit ist dabei relativ und nicht absolut zu verstehen. Relative Richtigkeit richtet sich nach der intersubjektiven Nachprüfbarkeit, d.h. bei Kenntnis der anzuwendenden Regeln muß die Aufstellung der Umwelterklärung nachvollziehbar sein.

\section{- Grundsatz der Willkürfreiheit:}

Dieser ergänzt den Richtigkeitsgrundsatz insofern als die Festlegung der Erfassungs- und Bewertungsmethoden nicht willkürlich erfolgen darf. Diese müssen vielmehr so ausgewählt werden, daß die Zweckerfüllung der Umwelterklärung bestmöglich erreicht wird.

\section{- Grundsatz der Vorsicht:}

Die Vornahme von vorsichtigen Bewertungen dient ebenfalls der Sicherung von zuverlässigen Angaben in der Umwelterklärung. Angesichts lückenhafter Kenntnisse und Erfahrungen über ökologische Folgewirkungen unternehmerischen Handelns, beispielsweise im Bereich der Toxikologie, sind hier im Zweifelsfall skeptische Annahmen zugrundezulegen.

\section{- Grundsatz der Klarheit:}

Die Angaben sind in $» k n a p p e r$, verständlicher Form «(9) vorzunehmen. Voraussetzung für eine sachgerechte Information ist die systematische und übersichtliche Darstellung der Sachverhalte.

\section{- Grundsatz der Kontinuität:}

Formelle (gleichbleibender Ausweis, Bezeichnungen und Gliederung) und materielle (gleichbleibende Erfassungs- und Bewertungsmethoden) Kontinuität bei der Erstellung der Erklärungen über die Berichtsperioden sichern zumindest intertemporale Vergleichbarkeit der Informationen für die Adressaten. Dies ist notwendige Voraussetzung, um die durch die Verordnung vorgegebene »kontinuierliche Verbesserung des betrieblichen Umweltschutzes«(10) beurteilen zu können.

Die angeführten Grundsätze sind nicht als abschließende anzusehen. In der Praxis müssen sich im Laufe der Zeit vielmehr zusätzliche Regeln für die Umweltberichterstattung herausbilden, die nicht nur auf die rechtlichen Vorga- 
ben begrenzt sein werden. Anzuführen sind beispielsweise die Notwendigkeit der Dialogorientierung und der Zielgruppengerechtheit (11).

Damit ist noch nichts gesagt über die spezifischen Inhalte der Erklärung. Die in Art. 5 Abs. 3 EU-Öko-Audit-Verordnung vorgenommene Aufzählung von Bestandteilen der Umwelterklärung ist als die Bestimmung von Mindestangaben zu festgelegten Themenbereichen zu verstehen. Dies wird durch die explizite Aufnahme der »sonstigen Faktoren « (12) als eine Art Auffangposten für Sachverhalte, die sich in den anderen genannten Punkten nicht finden lassen, bestärkt. Für die Konkretisierung der Einzelangaben, deren Bewertung und Aufbereitung liefern die Grundsätze ordnungsgemäßer Umweltberichterstattung wesentliche Hinweise.

Eine marktorientierte Kommunikation hat darüber hinaus das Interesse an Planungs- und Leistungskriterien zu berücksichtigen, die eine $\mathrm{Be}$ wertung des Unternehmenshandeln im Zeitablauf ermöglichen. In der betriebswirtschaftlichen Theorie findet man nicht ohne Grund die Einteilung der Kontrolle in Prämissen-, Ergebnis- und Verfahrens-/Verhaltenskontrolle. Erst unter Berücksichtigung aller drei Bestandteile ist eine aussagefähige Beurteilung der umweltorientierten Unternehmensführung möglich. Eine Beschränkung auf Teilaspekte, die etwa auf eine hinreichend spezifizierte Planungs- und Zielsetzungsbeschreibung oder auf nachprüfbare Ergebnisse im Hinblick auf die gesetzten Ziele verzichtet, erweist sich als Fortsetzung der traditionellen PR-Politik mit anderen Mitteln.

Juristische Zulässigkeit und Zielgruppengerechtheit der Umweltberichterstattung sind die zentralen Problemfacetten; imagefördernd und damit marktwirksam wird sie jedoch nur, wenn sie auf Resonanz im Mediensystem stößt.

\section{Öffentlichkeitswirksamkeit der Umwelterklärung}

Die Urteilsbildung über die betriebliche Umweltpolitik ist nur z.T. durch eigene Erfahrungen und direkte Kommunikation bestimmt. Umweltgefährdungen sind in mehrfacher Hinsicht fremdwissensabhängig: ob sie überhaupt vorhanden sind, wie risikobehaftet sie sind, in welcher Erscheinungsform sie auftreten, wie sie vermieden werden können usf. Ohne die Vermittlung der Medien bleiben Produkte ohne Folgen und Symptome ohne Ursachen (13). Bei einer empirischen Untersuchung $\mathrm{zu}$ der Frage nach den Gründen für umweltorientierte Entscheidungen bei Unternehmen werden erwartungsgemäß in erster Linie staatliche Vorgaben, aber schon an zweiter Stelle der öffentliche Druck als entscheidender Anstoßfaktor genannt (14). Die Medien bilden den zentralen »Kanalisator « (15) für die gesellschaftlichen Umweltanforderungen und werden lange vor den konkreten Kaufverhaltensänderungen wirksam.
Wenn Umwelterklärungen verhaltensprägend werden (und bleiben) sollen, dann ist die publizistische Aufmerksamkeit wohl eine notwendige Bedingung. Die Faktoren, die die Wahrnehmung des Mediensystems bestimmen, werden in der kommunikationswissenschaftlichen $\mathrm{Li}$ teratur als Nachrichtenwerte diskutiert (14):

\section{(1) Die Zeitdimension der}

Medienberichterstattung:

Aktualität und Innovationsgehalt

Medienberichterstattung zeichnet sich durch eine außergewöhnlich kurze Halbwertzeit aus. Nichts ist so alt wie die Nachrichten von gestern - dieser Journalistenspruch charakterisiert die medieninterne Nachrichtenobsoleszenz. Innovative Themen - wie derzeit Umweltberichterstattungen - werden entsprechend häufiger aufgegriffen. Umgekehrt droht die Gefahr des medieninternen Aufmerksamkeitsverlustes (weil ein Thema totgeschrieben ist) und dies weitgehend unabhängig davon, ob ein Problem gelöst, symbolisch angegangen oder nur »ausgesessen « ist. Die Zeit, bis eine Mediendebatte Ermüdungserscheinungen zeigt, ist knapp. Problematisch ist dabei, daß dann, wenn ein Thema einmal aus dem Blickfeld der Öffentlichkeit wieder verschwunden ist, ein Relaunch nur möglich ist, wenn eine neue Facette des Problems entdeckt wird, neue Regulierungskonzepte vorliegen, andere Akteure auftreten usf., d. h. wenn der Frage neue Medienaktualität verliehen werden kann. Gelingt dies nicht, ist das Thema »tot « und ein Relaunch bleibt zumindest schwierig, weil seine Geschichte eine Erneuerung blockiert. In dem daraus resultierenden Mangel an Kontinuität liegt eine wesentliche Bedrohung für Umwelterklärungen, wenn sie ihre Einführungsphase erst einmal hinter sich gelassen haben.

\section{(2) Zur Raumdimension der Nachrichtenwahl:} Bezug und Betroffenheit

Themen, die auf die lebensweltliche Erfahrung der Rezipienten Bezug nehmen, haben erfahrungsgemäß größere Chancen, aufgegriffen zu werden als andere. Die geographische, politische oder kulturelle Nähe eines Gegenstandes und damit die Möglichkeit, daß die Lebenspraxis des Publikums zumindest potentiell tangiert werden könnte, steigert die Veröffentlichungschancen enorm. Hier liegt der Stellenwert von Standorterklärungen begründet, die durch offensive lokale PR, aber auch durch Bürgerversammlungen u. ä. zum Gesprächsthema gemacht werden können.

\section{(3) Zur Sozialdimension der öffentlichen Meinung: Prominenz, Personifizierung und Emotionalität}

Ein zentraler Nachrichtenfaktor ist die Prominenz der beteiligten Akteure. Positionseliten bilden einen durchgehenden Betrachtungsfokus des Mediensystems. Hier gilt a priori die Vermutung öffentlicher Relevanz (17). Entsprechend schwierig werden es die derzeit noch überaus erfolgreichen mittelständischen Pionierunternehmen der Umweltberichterstattung haben, langfristig öffentliches Gehör zu finden. Problematisch für den öffentlichen Diskurs ist der Trend zur Personalisierung von Themen. So ermittelten mehrere Studien zur Themenberichterstattung bei Umweltkonflikten die Überrepräsentation der personalen Berichterstattung. Häufig beschränken sich Meldungen auf die Wiedergabe von Meinungen, so daß Hintergrundinformationen kaum Berücksichtigung finden. Damit steht die Konzentration der öffentlichen Aufmerksamkeit auf Kausalzusammenhänge und Verantwortlichkeiten im $\mathrm{Zu}$ sammenhang. Das Opfer-Täter-Bild ist ein verbreitetes Darstellungsmuster (18); es führt jedoch z. B. zur Ausblendung der alltäglichen und legalen Produktionsmuster und mindert damit generell den Nachrichtenwert der Umweltberichterstattung. Gleiches trifft auf den fehlenden emotionalen Charakter dieses Themas zu.

(4) Zur Sachdimension der Themenfindung: Kontroversität, Valenz, Quantifizierbarkeit und Visualität

Die Berichterstattung der Presse ist häufig dissensorientiert und polarisierend (19). Das Spektrum der Positionen wird auf ein handliches binäres Schema reduziert. Die Umweltproblematik bietet dazu vielfältigen $A n l a ß$, denn ohne Zweifel stehen sich z. B. in der Kernenergiefrage bis heute relativ unversöhnliche Gruppen gegenüber. Die Frage nach einer gesamtgesellschaftlich vertretbaren und ökonomisch tragfähigen Unternehmenspolitik läßt sich dagegen kaum in die Form einer einfachen Kontroverse bringen. In ähnlicher Richtung wirkt die gerade auch von Wirtschaftsseite häufig beklagte Überrepräsentanz von Negativmeldungen. Medien bevorzugen Außergewöhnliches, sei es ein Skandal oder ein überraschender Erfolg. (20) Damit wird gleichzeitig die kontinuierliche Auseinandersetzung mit einem Thema erschwert. Nicht die alltägliche Belastung, sondern schlaglichtartige Einzelfälle prägen das Bild, welches die Medien von den Umwelteffekten der Produktion vermitteln (21). Jüngstes Beispiel dafür war die geplante Versenkung der Brent Spar von Shell. Ein weitgehend unterschätzter Nachrichtenwert ist die Quantifizierbarkeit eines Ereignisses (22). Zahlreiche Phänomene rücken erst dann in den Blickpunkt der Öffentlichkeit, wenn sie durch Statistiken unterlegt werden können. Der Erfolg der Verschmutzungsranglisten in den USA erklärt sich wohl vor diesem Hintergrund. Mit dem Siegeszug der elektronischen Medien ist die steigende Bedeutung der Visualisierbarkeit als Nachrichtenfaktor verbunden (23). Die nüchternen Zahlen der Umwelterklärung vermögen insgesamt wenig »human touch « zu entwickeln.

Zusammengefaßt: Es wird deutlich, daß die Umweltberichterstattung als Medienthema derzeit vor allem aufgrund ihres Innovationsgrades aufgegriffen wird. Mit der zunehmenden Verbreitung durch die EU-Verordnung wird sie je- 
doch als PR-Instrument für die Unternehmen weitgehend an Bedeutung verlieren, wenn es nicht gelingt, durch neue Konzepte dem Thema jeweils Nachrichtenwert zu verleihen. Dabei fällt der Blick in erster Linie auf die vergleichende Quantifizierbarkeit der betrieblichen Umweltleistungen. Wenn überhaupt Chancen auf eine systematische, einzelfallübergreifende Debatte über die unternehmerische Umweltpolitik bestehen, dann durch leicht verständliche Betriebs- und Branchenvergleiche. Hier trifft sich zudem das öffentliche Interesse mit dem der ökologischen Pionierunternehmen.

\section{Perspektiven der Umweltkommunikation}

Es ist offensichtlich, daß die Veröffentlichung von Umwelterklärungen allein zunehmend weniger Aufmerksamkeit gewinnen wird. Sollte die Teilnahme an der EU-Verordnung in Deutschland erfolgreich diffundieren, dann wird die Zahl der Berichte in kurzer Zeit die Verarbeitungskapazität der kritischen Öffentlichkeit wie die der Medien übersteigen.

Eine Möglichkeit, Umwelterklärungen handlungswirksamer werden zu lassen, wäre die Einrichtung eines überbetrieblichen Informations-

1) Besonders prägnant diskutiert bei Marx, T.G., Strategic Planning for Public Affairs, in: Long-RangePlanning, Nr. 1/1990, S. 9-16

2) Vgl. Pfriem, R., Unternehmenspolitik in sozialökologischen Perspektiven, Marburg 1995, S. 336

3) Vgl. Kraemer, A., Die EG-Öko-Audit-Verordnung und ihr Umfeld in der Europäischen Umweltpolitik, in: Fichter, K. (Hg. ), EG-Öko-Audit-Verordnung, Mit Öko-Controlling zum zertifizierten Umweltmanagementsystem, SR 81/95 des IÖW

4) Vgl. 2, 4 Abs. 3, 4 a-e der 9. Verordnung zur Durchführung des Bundesimmissionsschutzgesetzes (Verordnung über das Genehmigungsverfahren vom 29.03.1992 (BGBI. I S. 1001), 2, 3 der Verordnung über das Verfahren bei der Genehmigung von Anlagen nach $\S 7$ des Atomgesetzes (Atomrechtliche Verfahrensverordnung-AtVfV) i.d.F. d. Bek. vom 03.02.1995 (BGBI. I, S. 180)

5) Anhang III Abschn. B. 4. EU-Ökoaudit-Verordnung

6) Vgl. bspw. Leffson, U., Die Grundsätze ordnungs- kreises zur Durchführung eines ökologischen Betriebsvergleichs. Auf freiwilliger Basis und unter Leitung eines umweltpolitisch ausgewiesenen Dritten, der für die Glaubwürdigkeit und die fachliche Integrität des Systems verantwortlich wäre, könnten sich Unternehmen zu einem »Reduktionsclub « zusammenschließen. Aufgaben könnten die Diskussion über betriebliche Umweltschutzziele, der Erfahrungsaustausch und schließlich die Durchführung von Betriebs- und Branchenvergleichen sein. Die Ergebnisse sollten durch offensive PR-Arbeit bekanntgemacht werden.

Die alternative Lösung, ein Ranking in Anlehnung an die »Top 500 Polluters« beim TRI in den USA, wäre wohl nur die zweitbeste Variante. Es ist zu erwarten, daß für externe Beobachter die Bewertung der betrieblichen Umweltdaten immer lückenhaft bleiben wird. Erinnert sei nur an Faktoren wie: Veränderungen der Fertigungstiefe (Out- oder Insourcing), des Auslastungsgrades, Produktinnovationen usw. Gleichwohl rechtfertigt das berechtigte öffentliche Interesse am Umwelthandeln der Betriebe auch vereinfachende Vergleiche.

Neben dieser Medienorientierung scheinen die größten Chancen der Umweltberichterstattung

mäßiger Buchführung, 7. Aufl., Düsseldorf 1987 7) Vgl. Förschle, G. / Mandler, U., Umwelterklärung, Umweltgutachter und Wirtschaftsprüfung, in: BFuP 6/1994, S. 524 f.

8) Vgl. Art. 4 Abs. 5) d) EU-Ökoaudit-Verordnung 9) Art. 5 Abs. 2 EU-Ökoaudit-Verordung

10) Art. 3. Pkt. a) EU-Ökoaudit-Verordnung

11) Vgl. Förderkreis Umwelt future e. V. (Hrsg. ), Umweltberichte -Umwelterklärungen, Hinweise zur Erstellung und Verbreitung, Osnabrück 1994, S. 17 ff. 12) Art. 5 Abs. 3 EU-Ökoaudit-Verordnung

13) Vgl. Beck, U., Risikogesellschaft, Frankfurt a. M. 1986, S. 70

14) Vgl. Coenenberg, A. G. et al., Unternehmenspolitik und Umweltschutz, in: ZfbF, Nr. 1/1994, S. 97 und Haßler, R., Öko-Rating - Ökologische Unternehmensbewertung als neues Informationsinstrument, Schriftenreihe zur ökologischen Kommunikation, Bd. 2, München 1994, S. 4

15) Meffert, H., Kirchgeorg, M., Der Einfluß von Ökologie und Marketing auf die Strategien, in: asw, in der Anlieger- und Mitarbeiterkommunikation zu liegen. Gerade für die Auseindersetzung mit den Anwohnern bedarf es jedoch weit mehr als der Veröffentlichung von Umwelterklärungen. Hier müssen von den Unternehmen Formen entwickelt und Anlässe iniiert werden, die das Interesse an umweltpolitischen Fortschritten immer wieder neu aktivieren. Nach allen bisher vorliegenden Praxiserfahrungen kommt den Beschäftigten beim Umwelt-Audit vornehmlich eine passive, auf die Befolgung von Verfahrensanweisungen kaprizierte Funktion zu. Die Erfahrungen der betriebswirtschaftlichen Organisationstheorie wie auch zahlreiche Fallstudien aus der Unternehmenspraxis verweisen jedoch darauf, daß ein Umweltmanagement als (alleiniger) Top-Down-Prozeß nur geringe Erfolgsaussichten aufweist. Arbeitnehmer und Management vor Ort wissen immer noch am besten, ob die heren Umwelterfolge, die in der Erklärung ausgelobt werden, freiwillige Umweltleistungen, Gratiseffekte betrieblicher Umstrukturierungen oder Ergebnis gesetzgeberischen Handelns sind.

Silke Hermann, Achim Spiller Eschborn

Sonderheft 10/1990, S. 42 und Schülein, J. A. et al., Manager und Ökologie, Opladen 1994, S. 187

16) Vgl. den Überblick bei Staab, J. F., Nachrichtenwert-Theorie, München 1990

17) Vgl. Schmitt-Beck, R., Über die Bedeutung der Massenmedien für soziale Bewegungen, in: Kölne Zeitschrift für Soziologie und Sozialpsychologie, Nr. 4/1990, S. $649 \mathrm{f}$.

18) Vgl. Wiedemann, P. M., Krisenkommunikation, 2. Aufl., Eschborn 1993, S. 37

19) Vgl. auch Ruhrmann, G., Risikokommunikation, in: Publizistik, Nr. 1/1992, S. 12

20) Vgl. auch Kepplinger, H. M., Weißbecker, H., Negativität als Nachrichtenideologie, in: Publizistik, Nr. $3 / 1991$, S. $330-342$

21) Vgl. Krämer, A., Ökologie und politische Öffentlichkeit, München 1986, S. 221

22) Vgl. Luhmann, N., Soziologische Aufklärung 5 Opladen 1990, S. $178 \mathrm{f}$

23) Vgl. Marcinkowski, F., Publizistik als autopoietisches System, Opladen 1993, S. 105

\section{Encouraging Improvements in Environmental Reporting - the Role of Award Schemes}

\begin{abstract}
Award schemes can play an important role in encouraging better corporate environmental and financial reporting. The environmental reporting award scheme described below is administered by the Chartered Association of Certified Accountants (ACCA), a United Kingdom accountancy body which is active in the field of environmental accounting research.
\end{abstract}

1994 was the fourth year of the annual Environmental Reporting Award Scheme (ERAS) sponsored by the Chartered Association of Certified Accountants (ACCA). The judges reported that greater clarity of focus and better environmental information systems have produced a steady improvement in the quality of company environmental reporting since 1991 when the award scheme was initiated. This had led to an increasing quality in reports submitted

The role which award schemes can play in encouraging better corporate behaviour (be it financial reporting or environmental management) has long been recognised. In the UK there are awards for the best public company set of financial statements, the best small company accounts, the best charity accounts, even the best accounts issued by a local authority. Similarly there are any number of awards recognising out- standing performance in the areas of environmental management. The underlying purpose is always the same - to encourage better reporting and to reward innovation. The ACCA Environmental Reporting Award scheme has a similar objective: »to stimulate and reward initiatives in environmental reporting «. The scheme is open to companies and public sector bodies throughout the European Union and covers both standalone environmental reports and environmental reporting through the medium of the annual corporate report. However, we are really only competent to deal with reports published in English although we did make efforts to translate one Dutch language and one German language entry for the 1994 scheme.

The ACCA environmental initiative has been given a warm reception in other countries. By the end of 1995 similar schemes will be running 
(c) 20I0 Authors; licensee IÖW and oekom verlag. This is an article distributed under the terms of the Creative Commons Attribution Non-Commercial No Derivates License (http://creativecommons.org/licenses/by-nc-nd/3.o/), which permits unrestricted use, distribution, and reproduction in any medium, provided the original work is properly cited. 